\title{
The Statistics of Gamma-Ray Burst Lensing
}

\author{
Scott A. Grossman and Michael A. Nowak \\ Canadian Institute for Theoretical Astrophysics \\ McLennan Labs, University of Toronto \\ 60 St. George Street \\ Toronto, Ontario M5S 1A7 \\ Canada \\ submitted to ApJ
}

\begin{abstract}
Possibly the only unambiguous verification that gamma-ray bursts (GRBs) are at cosmological distances would be the observation of multiple images of a gravitationally lensed burst. Each images would arrive at a different time, but exhibit identical light curves. We improve upon previous calculations of GRB lensing by using revised cosmological burst models based upon better knowledge of burst spectra, and we consider several sets of cosmological parameters. Only lensing by the known population of galaxies is considered, and we compute the lensing rate and amplification bias using three different lens models. Assuming that 800 bursts per year are above the detection threshold of the BATSE experiment on the Compton Gamma-Ray Observatory, we predict one lensing event every 1.5-25 years, with a median time delay between images of about 7 days. However, BATSE has an instrumental efficiency of about $12 \%$ for detecting double bursts, decreasing the detection rate to fewer than one per century for some cosmological parameters. This inefficiency can be overcome partially if cases of quadruple imaging by elliptical potentials are common, but simulations show that lensing by elliptical potentials enhances the detection rate by only about $20 \%$. We conclude that it is unlikely that the BATSE experiment will detect a lensed burst, but that a future experiment, which is either much more sensitive or has a much more efficient duty cycle, could.
\end{abstract}




\section{INTRODUCTION}

Data collected by the Burst and Transient Source Experiment (BATSE) on the Compton Gamma-Ray Observatory (CGRO) have generated interest in the cosmological origin scenario for gamma-ray bursts (GRBs) (see Blaes 1994 for a review of various scenarios). In particular, the isotropic distribution of bursts is not compatible with any known galactic distribution (disk or halo) of objects (Mao \& Paczyński 1992b). The GRBs must originate beyond $18 \mathrm{kpc}$ from the galactic center or be part of the solar system (e.g., in the Oort cloud) to have the required isotropy. Unfortunately, the flux distribution of bursts alone cannot distinguish among the local, extended halo, and cosmological possibilities (Lubin \& Wijers 1993).

Since BATSE's spatial resolution $\left(\sim 4^{\circ}\right)$ is insufficient to unambiguously associate individual bursts with individual high redshift galaxies, perhaps the only definitive proof of the cosmological origin of GRBs that BATSE could provide is a detection of a gravitational lensing event. A lensed GRB would show multiple images, but unlike quasars, they would not be identified as a spatially resolved pair (or quadruplet). Rather, they would appear as two bursts that arrive at different times and are located within the same positional error box on the sky. They will exhibit the same light curve, but probably will have different amplitudes.

Since the first suggestion that GRBs might be lensed (Paczyński 1986), several authors have presented detailed calculations of GRB lensing. Mao (1992b) considered lensing by the known population of galaxies. He found that GRBs should be lensed at a rate of about one per thousand, which is comparable to the rate of quasar lensing (Ostriker \& Vietri 1986). Other authors have considered lensing by a significant cosmological density of point masses (Mao 1992b; Blaes and Webster 1992). Computations of GRB microlensing (Paczyński 1987; Mao 1993) and even "femtolensing" (Gould 1992) have been considered. Narayan and Wallington (1992a) focused on what can be learned about the lens itself if a lensed burst is observed. Nemiroff et al. (1993) have examined the first 44 GRBs detected by BATSE for evidence of lensing. The absence of any lensed bursts in this sample is used to set rather loose constraints upon the density of $10^{6}-10^{8} M_{\odot}$ objects in the universe.

Considering the predicted rate of GRB lensing, it is not surprising that no lensing events were found in the small sample examined by Nemiroff et al. (1993). Indeed, if only galaxies act as lenses, none should be present in the sample of more than 300 bursts catalogued so far. Furthermore, even if there were a lensing event in the catalogued data, Wambsganss (1993) has claimed that the amount of noise present in the BATSE light curves may overwhelm any hope of identifying it. Nowak and Grossman (1994) find that this is excessively pessimistic. They conclude that a large fraction of bursts that are bright enough to trigger the BATSE detector ( $5.5 \sigma$ above the background noise) are also bright enough to have light curves that can be distinguished from other distinct bursts. Since previous calculations suggest that in a few more years of data there should be one lensing event if GRBs are cosmological, searching for lensed bursts may be an exercise with a potentially large return.

The purpose of this paper is to revise and expand upon the computations of lensing

by the known population of galaxies. In particular, we find a significantly lower lensing 


\section{$2 \quad G R B$ Lensing}

rate than did Mao (1992b), for two important reasons. First, he used a cosmological burst model where bursts can be seen to redshifts farther than are likely. According to recent models using improved burst spectra (Fenimore et al. 1993; Tamblyn \& Melia 1993, hereafter TM93), bursts are not likely to be seen beyond $z \sim 1$, whereas Mao (1992b) used a model where they can be seen to $z \sim 1.5$ (Mao and Paczyński 1992a). Second, Mao did not use the appropriate selection criterion for the occurrence of a lensing event, namely whether or not the second brightest burst is observable. In $\S 2$ we construct cosmological models of GRBs using the improved spectrum of TM93 for cosmologies $(\Omega, \Lambda)=(1.0,0)$, $(0.1,0),(0.1,0.9)$. In $\S 3$ we use these three cosmological models to compute the rate of observable double bursts, using the population of known galaxies, modeled as singular isothermal spheres, as lenses. In addition we consider the lensing rate if the lenses are non-singular isothermal spheres or have finite mass and no dark, isothermal halos. The lensing rate is high enough that a lensing event has a significant chance of occurring during BATSE's lifetime. We discuss how BATSE's instrumental inefficiency for detecting lensing events defeats this moderately optimistic rate. In $\S 4$ we consider lensing by an elliptical potential, and derive from simulations the probability for multiple imaging with two, three, or four observable components. If four component bursts are sufficiently common, then the instrumental inefficiency can be partly overcome. We demonstrate that lens ellipticity enhances the detection rate of lensing events by a small amount. We summarize and discuss the implications of our results for the BATSE experiment and potential future experiments in $\S 5$.

\section{THE GRB POPULATION}

Analysis of the flux distribution of GRBs (Meegan et al. 1992) shows that the observed $\left\langle V / V_{\max }\right\rangle$ statistic (Schmidt 1968) is not compatible with a homogeneous distribution. (This statement presupposes that the flux decreases as the inverse square of the distance.) Although the $\left\langle V / V_{\max }\right\rangle$ statistic can be reconciled with a local population that is absent at great distances, the observed GRB isotropy is inconsistent with a local galactic halo population (unless the core radius of the distribution is anomalously large, Mao \& Paczyński 1992b) and with a local population associated with the Oort cloud, Maoz 1993, Clarke et al. 1994). Another possibility is that the inverse square law does not hold, as would be the case if bursts are at cosmological distances $(z \gtrsim 0.3)$ such that the curvature of the universe is important. Several authors (Dermer 1992; Mao \& Paczyński 1992a; Piran 1992; Wickramasinghe et al. 1993; Fenimore et al. 1993; TM93) have shown that such departures from Euclidean geometry can account for the GRB flux distribution. The observation that fainter bursts have longer duration (Norris et al. 1994) is consistent with cosmological time dilation of distant bursts, and supports the cosmological scenario.

We assume GRBs originate at cosmological distances, and that the population can be described by standard candle luminosity $L_{0}$ and a constant burst rate per comoving volume $n_{0}$. The maximum distance to which a burst can be seen measures the departure from Euclidean geometry, and thus, $L_{0}$ is chosen to reproduce the observed $\left\langle V / V_{\max }\right\rangle$. Based on 207 bursts in the BATSE burst catalog (Fishman et al. 1992) up to March 5, 
1992 (overwrites are excluded), we compute $\left\langle V / V_{\max }\right\rangle=0.32$, in agreement with that from the slightly smaller sample in Meegan et al. (1992). The burst rate per unit volume $n_{0}$ is chosen to reproduce the observed burst rate, $N \approx 800 \mathrm{yr}^{-1}$.

The details of this calculation depend on the GRB spectrum. We adopt the double power-law model of TM93, which is based on the analysis of burst spectra by Band et al. (1993). The photon spectrum (i.e., photons per unit time per unit energy) is described by

$$
S_{E}(E)= \begin{cases}A E^{\alpha_{1}-1}, & E \leq E_{b} \\ A E_{b}^{\alpha_{1}-\alpha_{2}} E^{\alpha_{2}-1}, & E \geq E_{b}\end{cases}
$$

where $E_{b}=300 \mathrm{keV}$ is the break in the power-law slope. The power law exponents are $\alpha_{1}=1, \alpha_{2}=-1$. The coefficient $A$ is calibrated by equating the energy emitted in the bandpass $50-300 \mathrm{keV}$ (the bandpass in which BATSE bursts are observed) to the restframe luminosity, $L_{0}$.

The photon flux observed in the $50-300 \mathrm{keV}$ bandpass depends on the distance to the burst, measured by its redshift $z_{\mathrm{s}}$, and is given by

$$
\phi\left(z_{\mathrm{s}}\right)=\frac{S\left(z_{\mathrm{s}}\right)}{4 \pi d_{L}\left(z_{\mathrm{s}}\right)^{2}}
$$

where $d_{L}\left(z_{\mathrm{s}}\right)$ is the luminosity distance and $S\left(z_{\mathrm{s}}\right)$ is the K-corrected photon luminosity given by

$$
S\left(z_{\mathrm{s}}\right)=\int_{50 \mathrm{keV}}^{300 \mathrm{keV}} S_{E}\left[\left(1+z_{\mathrm{s}}\right) E\right] d E
$$

that is, the photon emission rate corrected for the redshifted bandpass. Evaluating this integral, we find

$$
S\left(z_{\mathrm{s}}\right)= \begin{cases}L_{0} 4.3 \times 10^{6}\left(1+z_{\mathrm{s}}\right)^{-1}\left[2-\left(1+z_{\mathrm{s}}\right)^{-1}-\frac{1}{6}\left(1+z_{\mathrm{s}}\right)\right], & z_{\mathrm{s}} \leq 5 \\ L_{0} 4.3 \times 10^{6}\left(1+z_{\mathrm{s}}\right)^{-2} 5, & z_{\mathrm{s}} \geq 5\end{cases}
$$

where $L_{0}$ is measured in $\mathrm{erg} \mathrm{s}^{-1}$. Bursts with fluxes that are $5.5 \sigma$ above background in either the $64 \mathrm{~ms}, 256 \mathrm{~ms}$, or $1024 \mathrm{~ms}$ time bins trigger BATSE. The $1024 \mathrm{~ms}$ trigger threshold is most sensitive, so we adopt its flux limit, $\phi_{\min }=0.3$ photon s${ }^{-1} \mathrm{~cm}^{-2}$ for $\gtrsim 50 \%$ detection probability (Fishman et al. 1992). The flux limit $\phi_{\min }$ defines the maximum redshift $z_{\max }$ out to which bursts can be seen.

The rate at which we observe GRBs that originate in redshift interval $\left[z_{\mathrm{s}}, z_{\mathrm{s}}+d z_{\mathrm{s}}\right]$ is given by

$$
\frac{d N}{d z_{\mathrm{s}}}=\frac{n_{0}}{\left(1+z_{\mathrm{s}}\right)} \frac{d V}{d z_{\mathrm{s}}}
$$

The factor $1+z_{\mathrm{s}}$ comes from the gravitational time dilation of the burst rate, and $d V / d z_{\mathrm{s}}$ is the comoving volume element. Using equations (2) and (5), we can calculate how 


\section{$4 \quad$ GRB Lensing}

many bursts are observed with flux $\phi$. The theoretically predicted $\left\langle V / V_{\max }\right\rangle$ for this flux distribution is given by

$$
\left\langle\frac{V}{V_{\max }}\right\rangle=\left\langle\left(\frac{\phi}{\phi_{\min }}\right)^{-3 / 2}\right\rangle=\frac{\int_{0}^{z_{\max }}\left(\phi\left(z_{\mathrm{s}}\right) / \phi_{\min }\right)^{-3 / 2}\left(d N / d z_{s}\right) d z_{\mathrm{s}}}{\int_{0}^{z_{\max }}\left(d N / d z_{\mathrm{s}}\right) d z_{\mathrm{s}}} .
$$

The only missing ingredient required to carry out this computation is the specific choice of a cosmological model for the comoving volume element and the luminosity distance $d_{L}$. We will consider three Friedmann-Robertson-Walker cosmologies described by density and cosmological constant parameters $(\Omega, \Lambda)$ given by $(1.0,0),(0.1,0),(0.1,0.9)$. Cosmologies with cosmological constant much more than 0.9 probably already are excluded by the quasar lensing rate (Fukugita et al. 1992). The proper, luminosity, and angular distances are given by ( $c f$. Carrol, Press, \& Turner 1992)

$$
\begin{gathered}
d_{M}\left(z_{\mathrm{s}}\right)=\frac{c}{H_{0}} \int_{0}^{z_{\mathrm{s}}}\left[(1+z)^{2}(1+\Omega z)-z(2+z) \Lambda\right]^{-1 / 2} d z \\
d_{L}=\left(1+z_{\mathrm{s}}\right) d_{M} \\
d_{A}=d_{M} /\left(1+z_{\mathrm{s}}\right) .
\end{gathered}
$$

In general, these distances must be computed numerically. The angular distance between two redshifts $d_{A}\left(z_{1}, z_{\mathrm{s}}\right)$ can be found by replacing the lower bound of integration in equation (7a) with the lower redshift. The comoving volume element is given by

$$
\frac{d V}{d z_{\mathrm{s}}}=4 \pi d_{M}^{2} \frac{d}{d z_{\mathrm{s}}} d_{M}=4 \pi d_{M}^{2} \frac{c}{H_{0}}\left[\left(1+z_{\mathrm{s}}\right)^{2}\left(1+\Omega z_{\mathrm{s}}\right)-z_{\mathrm{s}}\left(2+z_{\mathrm{s}}\right) \Lambda\right]^{-1 / 2} .
$$

The quantitative effects of using an alternative, clumpy universe cosmology (Dyer \& Roeder 1973) are negligible for the moderate redshifts $z_{\mathrm{s}} \sim 1$ out to which bursts can be seen (Fukugita et al. 1992).

The values of $L_{0}, n_{0}$, and $z_{\max }$ that reproduce the observed $\left\langle V / V_{\max }\right\rangle$ and burst rate are listed in Table 1. The parameters for $\Omega=1, \Lambda=0$ are in approximate agreement with those of Fenimore et al. (1993) (presumably the differences are a consequence of using a different spectrum). Our $\Omega=0.1, \Lambda=0.9$ model also is consistent with that of Piran (1992), but no prior results are available for the $\Omega=0.1, \Lambda=0$ cosmology. We expect that a more careful analysis, for example including PVO data, would modify the results only slightly. We have not been more precise since burst modeling is not the aim of this paper, but is only one ingredient in the lensing calculation to follow.

In Figure 1, the redshift distributions of bursts are shown. In fact, for each cosmology two curves are drawn. One curve is the computation of equation (5), and the second is the same computation, but showing the incompleteness as fluxes approach and fall below $\phi_{\min }$. We have found that the data in the BATSE efficiency table for $1024 \mathrm{~ms}$ triggers (Fishman et al. 1992) is fit very well by the function

$$
\epsilon(\phi)= \begin{cases}0, & \phi \leq \phi_{0} \\ 1-e^{-\left(\phi-\phi_{0}\right) / \alpha}, & \phi \geq \phi_{0}\end{cases}
$$


with $\phi_{0}=0.21$ photon $\mathrm{s}^{-1} \mathrm{~cm}^{-2}$ and $\alpha=0.105$ photon $\mathrm{s}^{-1} \mathrm{~cm}^{-2}$. We use equation (9) as the detection efficiency in this and all subsequent calculations. The dotted vertical lines are drawn at the values of $z_{\max }$, where by definition the incompleteness is $50 \%$. Note that some bursts originating at $z_{\mathrm{s}}>z_{\max }$ will be seen when Poisson uncertainty in the photon rate brings the measured flux above $\phi_{\min }$.

Band (1992, see also Petrosian 1993) has argued that failure to account for incompleteness near the flux threshold invalidates many comparisons between the observed and predicted $\left\langle V / V_{\max }\right\rangle$. We test the sensitivity of our parameters to incompleteness by including the detection efficiency function, equation (9) in our calculation of $\left\langle\frac{V}{V_{\max }}\right\rangle$. Multiplying the top and bottom integrands of equation 6by the detection efficiency and integrating the bursts between $z=0$ and $z=z_{\max }$, we find $\left\langle V / V_{\max }\right\rangle=0.27$ instead of 0.32 for our $\Omega=1, \Lambda=0$ parameters. Furthermore, our and other models (e.g., TM93) reproduce the observed $\left\langle V / V_{\max }\right\rangle$ at thresholds well above the regime where incompleteness is relevant, but where cosmological effects are still important. Whether or not we include this incompleteness should modify the details of our cosmological model only slightly.

\section{THE RATE OF LENSING BY GALAXIES}

In this section we consider lensing by circular lens models. (See Blandford \& Narayan 1992, Narayan \& Wallington 1992b for reviews of the physics of lensing.) Such lenses alway produce at least one image of magnification $M_{1}$ greater than unity. This image is usually the brightest and most distant from the center of the lens. If there are multiple images, a second image with magnification $M_{2}$ appears on the opposite side of the lens. This image can be very bright or very faint, depending on the lens model and the position of the source with respect to the lens. If the lens has a non-singular core, there can be a third image of magnification $M_{3}$ in the core. This image is almost always very faint.

The differential lensing rate of GRBs is given by

$$
\frac{d N_{\mathrm{l}}}{d z_{\mathrm{s}}}=\frac{d N}{d z_{s}} \frac{\sigma_{\mathrm{tot}}\left(z_{\mathrm{s}}\right)}{4 \pi},
$$

where $\sigma_{\text {tot }}\left(z_{\mathrm{s}}\right)$ is the total cross section of all lenses in the sky to produce an observable multiple burst from a source in the interval $\left[z_{\mathrm{s}}, z_{\mathrm{s}}+d z_{\mathrm{s}}\right]$. If galaxies are the lensing masses, we can write the total cross section as

$$
\sigma_{\text {tot }}\left(z_{\mathrm{s}}\right)=\int_{0}^{z_{\mathrm{s}}} d z_{1} \frac{d V}{d z_{1}} \int_{0}^{\infty} d L \Phi(L) \sigma\left(z_{1}, z_{\mathrm{s}}, L\right) .
$$

The cross section for a single galaxy, $\sigma\left(z_{1}, z_{\mathrm{s}}, L\right)$, depends on the lens mass, which is directly related to lens luminosity. Weighting these cross sections by the galaxy luminosity function $\Phi(L)$, the inner integral sums over all lens masses at a single lens redshift $z_{1}$, and the outer integral sums over all the lensing galaxies at each lens redshift. The lens population is characterized by the Schecter luminosity function,

$$
\Phi(L) d L=\Phi^{*} e^{-L / L_{*}}\left(\frac{L}{L_{*}}\right)^{\beta} \frac{d L}{L_{*}},
$$




\section{$6 \quad$ GRB Lensing}

with parameters $\Phi^{*}=1.56 \times 10^{-2} h_{100}^{3} \mathrm{Mpc}^{-3}, \beta=-1.1$, as assumed in Fukugita and Turner (1991, hereafter FT91). The dimensionless Hubble constant is defined by $h_{100}=$ $H_{0} / 100 \mathrm{~km} \mathrm{~s}^{-1} \mathrm{Mpc}^{-1}$.

The lensing cross section of a single galaxy of luminosity $L$ to produce an observable multiple burst is

$$
\sigma\left(z_{1}, z_{\mathrm{s}}, L\right)=\int_{0}^{r_{\mathrm{crit}}} d r_{\mathrm{s}} 2 \pi r_{\mathrm{s}} \epsilon\left[M_{2}\left(r_{\mathrm{s}}\right) \phi\left(z_{\mathrm{s}}\right)\right]
$$

The variable $r_{\mathrm{s}}$ is the angular distance of a source to the lens axis. We have assumed we can relate the luminosity of a lens to its lens parameters. The value of $r_{\text {crit }}$, the outermost source radius that gives multiple images, depends on the particular lens model. Whether or not multiple images will be seen depends on the magnification $M_{2}$ of the fainter image with photon flux $M_{2} \phi\left(z_{\mathrm{s}}\right)$. If the detection efficiency $\epsilon\left[M_{2} \phi\left(z_{\mathrm{s}}\right)\right]$ is below unity, the effective cross section is reduced below its maximum value $\pi r_{\text {crit }}^{2}$. (We note that Mao, 1992b assumed that the full isothermal cross section contributed. Blaes \& Webster, 1992 did use the right selection criterion in their computation for point lenses.) Depending on the source position $r_{\mathrm{s}}, M_{2}$ can be greater or less than unity. If $M_{2}$ is greater than unity, it is possible to see bursts beyond $z_{\max }$, but if it is less than unity, bursts may have to be considerably closer than $z_{\max }$ for two images to be detectable.

In addition to the rate at which GRBs are lensed, we would like to know the characteristic time interval between the arrival of two images. The distribution of the time delay $\Delta t$ between two bursts, which was computed first by Mao (1992b), is given by the formula

$$
\frac{d N_{l}}{d \Delta t}=\int_{0}^{\infty} d z_{\mathrm{s}} \frac{d N}{d z_{\mathrm{s}}} \int_{0}^{z_{\mathrm{s}}} d z_{1} \frac{d V}{d z_{1}} \int_{0}^{\infty} d L \Phi(L) \frac{\partial \sigma\left(z_{1}, z_{\mathrm{s}}, L\right)}{\partial \Delta t} \epsilon\left[M_{2}\left(r_{\mathrm{s}}\right) \phi\left(z_{\mathrm{s}}\right)\right]
$$

For any particular lens model, the time delay $\Delta t$ can be related to the source position $r_{\mathrm{s}}$, so that the differential cross section is

$$
\frac{\partial \sigma}{\partial \Delta t}=\frac{2 \pi r_{\mathrm{s}} d r_{\mathrm{s}}}{\frac{\partial \Delta t}{\partial r_{\mathrm{s}}} d r_{\mathrm{s}}}
$$

\subsection{The Singular Isothermal Sphere (SIS)}

The singular isothermal lens (SIS) produces two images of the source whenever the source position lies inside the Einstein radius, $r_{\mathrm{S}}<r_{E}$, given by

$$
r_{\mathrm{crit}}=r_{E}=4 \pi \frac{v^{2}}{c^{2}} \frac{d_{A}\left(z_{1}, z_{\mathrm{s}}\right)}{d_{A}\left(0, z_{s}\right)}
$$

where $v$ is the line-of-sight velocity dispersion of the lensing galaxy. It is related to the lens luminosity via the Faber-Jackson or Tully-Fisher relations,

$$
\frac{L}{L_{*}}=\left(\frac{v}{v_{*}}\right)^{n}
$$


Following FT91, the lens population is assumed to be comprised of E, S0, and S galaxies in the proportion $12 \%, 19 \%$, and $69 \%$, with corresponding parameters $v_{*}=225 \sqrt{3 / 2} \mathrm{~km} \mathrm{~s}^{-1}$, $n=4$ for Es, $v_{*}=206 \sqrt{3 / 2} \mathrm{~km} \mathrm{~s}^{-1}, n=4$ for S0s, and $v_{*}=144 \mathrm{~km} \mathrm{~s}^{-1}, n=2.6$ for Ss. The factor of $\sqrt{3 / 2}$ in the velocity dispersion of Es and S0s accounts for isothermal dark halos surrounding more compact mass distributions (Turner, Ostriker, \& Gott 1984; FT91).

In the SIS lens, the magnification $M_{2}$ is related to the source position by

$$
M_{2}=r_{E} / r_{\mathrm{s}}-1 \text {. }
$$

For $r_{\mathrm{s}}>r_{E} / 2$ (three quarters of the maximum cross section $\pi r_{E}^{2}$ ), $M_{2}$ is less than unity, and $M_{2}$ goes to zero as $r_{\mathrm{s}}$ approaches $r_{E}$, making the second image unobservable. In no case does a galaxy contribute the maximum cross section $\pi r_{E}^{2}$.

Combining equations (10)-(13), (16)-(18) and evaluating the integrals numerically, we compute the lens rate as a function of source redshift $z_{\mathrm{s}}$ for each cosmology. The results are shown in Figure 2. For "standard" cosmological values $\Omega=1, \Lambda=0$, we get a total multiple burst rate of $0.09 \mathrm{yr}^{-1}$. This is quite a bit smaller than the value of $1.1 \mathrm{yr}^{-1}$ predicted by Mao (1992b), both because he adopted a GRB model in which unlensed bursts could be seen out to redshifts $z_{\max } \sim 1.5$ and because he used the maximum isothermal cross section. As expected (cf. Carrol, Press, \& Turner 1992; Turner 1990; Fukugita, Futamase, \& Kasai 1990), the finite $\Lambda$ model gives the largest lensing rate, about 7 times larger than the standard model.

The amplification bias is the ratio of the number of lensed bursts to the lensing rate computed as if bursts were not magnified. That is, it is the increase in the number of lensing events because sources intrinsically fainter than the flux threshold $\phi_{\min }$ are counted. We compute the hypothetical, no magnification lensing rate by replacing the detection frequency in equation (13) with $\epsilon\left[\phi\left(z_{\mathrm{s}}\right)\right]$, i.e., assuming the magnification is unity. The cumulative burst rates and the amplification biases computed for the various cosmologies are presented in Table 2. The amplification biases are smaller than for quasar lensing. Two factors contribute to this. One is that the observed GRB flux distribution is not as steep, so that the increase in the number of observable sources resulting from amplification bias is not as large. The other, equally important factor is that amplification bias is computed using the magnification probability of the fainter second image. This differs from most computations of quasar lensing, where the magnification probability is computed either for the brighter image or for the total magnification (e.g., Turner, Ostriker \& Gott 1984; FT91; but see Sasaki \& Takahara 1993 for an exception). In either case, the magnifications of the quasars are always greater than unity, so that there is no loss of cross section for sources at $z<z_{\max }$. For GRBs, however, we find a bias $B \sim 1$ for the $\Omega=1, \Lambda=0$ model. Even though lensed GRBs can be seen to a somewhat higher redshift than unlensed bursts (compare Figs. 1 and 2), the loss of lensing cross section for small $M_{2}$ almost perfectly offsets this increase. That is, even for sources at low redshifts, the second image will be too faint to see for some source positions that yield double images. In fact, biases can be less than unity (Sasaki \& Takahara 1993), as in the $\Omega=0.1, \Lambda=0$ model.

Even if our models of GRBs are more or less correct, reproducing the right $z_{\max }$, there are uncertainties in the lens rate resulting from uncertainties in the lens parameters. In 


\section{GRB Lensing}

particular, since the lens rate varies as $N_{l} \sim \Phi^{*} v^{4} \sim \Phi^{*} v_{*}^{4}\left(L / L_{*}\right)^{4 / n}$, the $10 \%$ uncertainty in $L_{*}, 22 \%$ uncertainty in $\Phi^{*}$, and $10 \%$ uncertainty in $v_{*}$ (e.g., FT91; Mao 1992a) give nearly a 50\% uncertainty in the computed lensing rates. Using a different observational luminosity function, Wallington and Narayan (1993) find a lensing rate about $40 \%$ larger than that given by the FT91 parameters.

We next compute the statistical distribution of time delays. The time delay of a pair of images in a lens of Einstein radius $r_{E}$ is given by

$$
\Delta t=f\left(z_{1}, z_{\mathrm{s}}, r_{E}\right) r_{\mathrm{s}}, \quad r_{\mathrm{s}}<r_{E}
$$

where we define the function

$$
f\left(z_{1}, z_{\mathrm{s}}, r_{E}\right)=2\left(1+z_{1}\right) \frac{d_{A}\left(0, z_{1}\right)}{c} \frac{d_{A}\left(0, z_{\mathrm{s}}\right)}{d_{A}\left(z_{1}, z_{\mathrm{s}}\right)} r_{E}
$$

Then the differential cross section (eq. 15) is

$$
\frac{\partial \sigma}{\partial \Delta t}=\frac{2 \pi \Delta t}{f^{2}} .
$$

The corresponding source position is given by $r_{\mathrm{s}}(\Delta t)=\Delta t / f$, and hence the magnification $M_{2}$ of the second image can be calculated by equation (18).

Substituting equation (21) into equation (14), we compute the distribution of time delays. The results are shown in Figure 3. The curves are divergent at small time delay due to the infinite number of low luminosity sources predicted by the luminosity function (eq. 12), but the total lensing rate, i.e., the integral under the curves, is finite and gives, of course, precisely the same cumulative lensing rate as the calculation for Figure 2 . The median time delay is about $0.02 h_{100}^{-1} \mathrm{yr}$ for the two $\Lambda=0$ cosmologies and about $0.04 h_{100}^{-1} \mathrm{yr}$ for the $\Omega=0.1, \Lambda=0.9$ cosmology.

\subsection{The Non-Singular Isothermal Sphere (NSIS)}

Realistic galaxy lenses probably have finite core radii. As is well-known, circular lenses with finite core radii can, in addition to the pair of images of a singular lens, produce a third image near the core of the lens. We adopt the usual non-singular isothermal lens model (NSIS, e.g., Hinshaw \& Krauss 1987), for which we can calculate image positions and magnifications for any source position. The only difference between this calculation and the previous one is that we have replaced equation (18) with the appropriate relation for an NSIS lens.

In previous investigations of quasar lensing (FT91; Mao 1992a), the importance of the core radius in galaxy lenses was based upon the observations of 42 elliptical galaxies by Lauer (1985). Lauer observed that 14 ellipticals had resolved cores, with half light radii that approximately follow the relation

$$
\frac{r_{1 / 2}}{r_{1 / 2}^{*}} \approx\left(\frac{L}{L_{*}}\right)^{1.2}
$$


where $r_{1 / 2}^{*} d_{A}\left(z_{1}\right)=0.16 \mathrm{kpc}$. The core radius, $r_{\mathrm{c}}$, of the lens model is related to the half light radius by $r_{1 / 2}=\sqrt{3} r_{c}$. The remaining ellipticals had unresolved cores, so that we may regard the above values of $r_{1 / 2}$ as upper limits. We assume all types of galaxies (E, S0, S) have core radii following the scaling of equation (22). The resulting lensing rates are reduced by about a factor of two, and this may be an overestimate if galaxy cores are often smaller than the above prediction. The lensing rates and amplification biases for the NSIS model are listed in Table 2.

To compute how often the third core image is seen, we simply replace the magnification $M_{2}$ in the detection efficiency computation with the magnification of the core image $M_{3}$. We find that the third image will be observable in fewer than $1 \%$ of the double bursts. The third image will be visible only in the very rare, very luminous lenses where the core radius is large and the central image is only moderately demagnified.

\subsection{A Finite Mass Lens Model (QDEV)}

If galaxies, particularly ellipticals, do not have massive isothermal halos, lensing computations based on a density profile containing finite mass may be more appropriate. For this analysis we adopt the quasi-de Vaucouleurs (QDEV) profile of Grossman and Saha (1994). This lens model is characterized by the deflection law

$$
\alpha(r)=\frac{4 G M}{d_{A}\left(0, z_{1}\right) c^{2}} \frac{r}{\left(r_{\mathrm{c}}+r\right)^{2}} .
$$

This deflection law closely resembles the true de Vaucouleurs deflection law (Sanitt 1976) if the core radius $r_{\mathrm{c}}=0.4 r_{e}$, where $r_{e}$ is the half mass radius, but it is simpler to use in numerical calculations.

We use the relations of Maoz and Rix (1993) to relate the luminosity of a lens to the lens parameters, core radius and mass. These relations are

$$
r_{\mathrm{c}}=0.4 r_{e}=0.4 r_{e}^{*}\left(\frac{L}{L_{*}}\right)^{a}
$$

and

$$
\frac{M}{L}=\left(\frac{M}{L}\right)^{*}\left(\frac{L}{L_{*}}\right)^{b},
$$

where the parameters are $r_{e}^{*}=4 \mathrm{kpc},(M / L)^{*}=10, a=1.2, b=0.25$. This lens can give three images, and as with the NSIS model, we are able to compute their positions and magnifications for any source position. Using the magnification of the second image $M_{2}$ in the calculation of detection efficiency, we find the lensing rate of this model.

The lensing rate of GRBs, assuming all types of galaxy lenses can be modeled in this way, is given in Table 2 . These rates are generally about half the SIS rate. The third core image is bright enough to be seen only about $0.1 \%$ of the time. The amplification biases are quite small. This is essentially because the cross section for multiple imaging is much larger than for the SIS lens (approaching the formally infinite cross section for a point 
lens), but the cross section for two images to be bright enough to observe is similar to the SIS cross section. Although lensing permits sources somewhat fainter and more distant than $z_{\max }$ to be observed, the loss of lensing cross section associated with small values of $M_{2}$ more than offsets this increase.

The lensing rate of this model scales as $N_{l} \sim \Phi^{*}(M / L) L \sim \Phi^{*}(M / L)^{*} / L_{*}^{b}$. The $22 \%$, $20 \%$, and $10 \%$ uncertainties in $\Phi^{*},(M / L)^{*}$, and $L_{*}$ (FT91; Maoz \& Rix 1993) combine to give about a $30 \%$ uncertainty in the computed lens rate.

\subsection{The BATSE Detection Rate}

The most pessimistic rate of observable double bursts in Table 2 is one every 25 years, while the most optimistic is one every 1.5 years. A standard $\Omega=1, \Lambda=0$ cosmology gives one every 10 years. Since GRO is likely to operate for at least several years, the odds are significant that there will be a lensed burst during this period if GRBs are cosmological.

Unfortunately, BATSE is inefficient at detecting bursts. Due to Earth blockage resulting from CGRO's low orbit $(450 \mathrm{~km}$, Gehrels et al. 1993), BATSE only "sees" about $2.6 \pi$ steradians of the sky at one time, and the detectors have a duty cycle of only about $55 \%$ (Fenimore 1993), giving a probability of 34\% (Fishman et al. 1992) to see any single burst. Since the time delay between lensed bursts is typically much longer than the orbital period of the satellite (many days compared to 100 minutes), the position of the satellite in orbit is essentially random with respect to burst arrival times. Thus, the odds of seeing both components of a double burst are $34 \% \times 34 \%=12 \%$. In view of this instrumental inefficiency, if lenses are of the simple sort described above, it is very unlikely that multiple bursts will be seen. This instrumental inefficiency generally has been ignored in previous GRB lensing papers (an exception being Mao, 1992b), resulting in undue optimism (e.g., Blaes 1994) about the possibility of observing a lensing event in the lifetime of BATSE.

\section{LENSING BY AN ELLIPTICAL POTENTIAL}

About half of known multiply-imaged quasars show four, rather than two, bright images (e.g., Blandford \& Narayan 1992). The simplest lens that can have such an image configuration is one with an elliptical gravitational potential. (See Narayan \& Grossman, 1989 for a review of caustics, critical lines, and images in an elliptical lens.) If the requirement to observe a lensing event is for BATSE to detect two or more images of a quadruple burst, the instrumental detection efficiency may be much higher than the $12 \%$ of a double burst. In general, the probability of detecting two or more images out of $n$ that are bright enough to be seen is given by the binomial probability

$$
P(\geq 2 \mid n)=\sum_{i=2}^{n}\left(\begin{array}{c}
n \\
i
\end{array}\right) p^{i}(1-p)^{n-i}
$$

where $p=34 \%$. If only two images are bright enough to be seen, this gives $P(\geq 2 \mid 2)=12 \%$; if three, $P(\geq 2 \mid 3)=27 \%$; and if four, $P(\geq 2 \mid 4)=42 \%$. Thus, if quadruple images are 
sufficiently common, then the chance of detecting a lensing event may be better than predicted for circular lens models.

To investigate the importance of elliptical lenses in GRB lensing, we model galaxy lenses by elliptical generalizations of the SIS potential, as used in the statistical lensing of quasars (Wallington \& Narayan 1993). The scaled two-dimensional potential $\psi$ (defined such that the deflection angle is $\alpha=-\nabla \psi$ ) is given by

$$
\psi(x, y)=r_{E}\left[(1+\epsilon) x^{2}+(1-\epsilon) y^{2}\right]^{1 / 2}
$$

where $\epsilon$ measures the lens ellipticity. We derive the statistics of elliptical lenses using a Monte Carlo simulation, where we randomly distribute isothermal lenses between the observer and source at $z_{\mathrm{s}}$ according to their luminosity distribution given by equation (12) and spatial distribution given by the comoving volume element. At each source redshift $z_{\mathrm{s}}$ at 0.2 intervals, we randomly place 10,000 sources within a circle of radius 1.3 times the Einstein radius (since we are interested only in potentially observable multiple bursts). The resulting statistical contribution to the time delay distribution of a pair of lensed bursts has a weight proportional to the area of the Einstein ring.

All the images of a single source are located using an efficient grid search method, where we identify rough image locations on a coarse grid and iteratively subdivide to higher resolution only those grid cells which may contain an image. Each iteration increases the resolution by a factor of 10, and it goes 8 levels deep. Sometimes several neighboring pixels are marked as containing an image, so as a final step, we compute the $x$ and $y$ components of the lens equation $\left(e . g ., x_{s}=x-\alpha(x, y)\right)$ at the four corners of each pixel and keep only pixels where the source position is bounded by the four corner values. We use the four corner values of $x_{s}$ and $y_{s}$ to interpolate $x$ and $y$ to a refined image position.

The results for a lens of ellipticity $\epsilon=0.2$ are shown in Figure 4, where we plot as a bold line the ratio of the cross section of the elliptical lens for observable multiple imaging, $\sigma_{\epsilon}(2+)$, to the cross section of the circular lens for double imaging, $\sigma_{0}(2)$. The cross section $\sigma_{\epsilon}(2+)$ is divided into the contributions of two-image bursts, three-image bursts, and fourimage bursts, i.e., $\sigma_{\epsilon}(2+)=\sigma_{\epsilon}(2)+\sigma_{\epsilon}(3)+\sigma_{\epsilon}(4)$. Analyzing the data of Huchra et al. (1983), Wallington and Narayan (1993) show that few galaxies have ellipticities exceeding $\epsilon=0.3$. They find a mean ellipticity somewhat less than $\epsilon=0.2$, and thus, using $\epsilon=0.2$ in Figure 4 is roughly representative of a typical galaxy lens.

The caustic curves of an elliptical lens divide the source plane into regions capable of producing one, three, and five images. The five image region is bounded by a diamondshaped astroid, and the associated cross section for producing five images is $\sigma_{5}$. The highest magnification events are associated with sources near folds or cusps on the caustic. The three image region is entirely outside this astroid (as long as the core radius is small enough), and is bounded on the outside by an ellipse with mean radius $r_{E}$. The associated cross section is $\sigma_{3}$, and bright images associated with appear on opposite sides of the lens. For lenses with small core radius, the central image of a three or five image lens is highly demagnified, and since we use a singular lens, the central image is always absent. Thus, the $\sigma_{3}$ and sigma $_{5}$ cross sections produce two and four images, respectively. 


\section{GRB Lensing}

The elliptical lens produces fewer multiple imaging events with images above the detector threshold than does the circular lens. This is essentially because, unlike a circular lens which produces high magnification images in pairs, an elliptical lens can produce one (or more) bright image of a source near a cusp, while leaving its counter-image magnified much less (e.g., Narayan \& Grossman 1989). Highly magnified pairs or triplets of images are likely to be mergers across folds and cusps. Counter-images with lower magnification will not be seen at redshifts far beyond $z_{\max }$, and indeed there are essentially no quadruple images in Figure 4 beyond $z_{\mathrm{s}} \approx 1.2$.

For a lens with $\epsilon=0.2$ and small core radius such that the diamond caustic (defining the five-image region) lies entirely within the the radial caustic (bounding the three-image region), the three-image cross section $\sigma_{3}$ is greater than the five-image cross section $\sigma_{5}$ at low and moderate magnifications (Wallington \& Narayan 1993). Images and counterimages can be seen if a source is at low redshift, and, therefore, pairs of images dominate the multiple image cross section $\sigma(2+)$. The three-image cross section varies as $\sigma_{3} \sim M^{-2.5}$, while the five-image cross section varies as $\sigma_{5} \sim M^{-2}$, so that at large magnifications, the five-image cross section dominates over the three-image cross section. The transition to $\sigma_{5}$ dominance is at magnification $M \sim 10$ (Wallington \& Narayan 1993). Thus, three- and four-image bursts become increasingly more important at larger redshifts, where bursts must be magnified more just to be seen. (We associate both three- and four-image bursts with $\sigma_{5}$ since they arise mainly from cusp imaging. Two-image bursts are mainly associated with $\sigma_{3}$ when they are at low redshift. At high redshift, however, the double images are mergers across folds, rather than image/counter-image pairs, so that these pairs are also associated with $\sigma_{5}$.) However, due to the low magnification bias, very high magnification events are not very common, and three- and four-image bursts are never seen to dominate over two-image bursts, unlike quasar imaging.

Although the absolute rate of observable multiple imaging by an elliptical potential is reduced by factor $\sigma_{\epsilon}(2+) / \sigma_{0}(2)$, it is possible that the rate at which lensing events are detected is increased. The enhancement of the rate at which lensing events are detected is given by the multiple image cross sections, weighted by the BATSE detection probability, according to

$$
\frac{N_{\epsilon}}{N_{0}}=\frac{0.12 \sigma_{\epsilon}(2)+0.27 \sigma_{\epsilon}(3)+0.42 \sigma_{\epsilon}(4)}{0.12 \sigma_{0}(2)} .
$$

This ratio is drawn as the dashed line in Figure 4. At the low and intermediate redshifts that give quadruple bursts, the detection rate is enhanced above the circular lens rate. For the most probable source redshift of $z_{\mathrm{s}} \approx 0.8$ (see Fig. $2, \Omega=1, \Lambda=0$ curve), the detection rate is about $30 \%$ larger than for a circular lens. If $\epsilon=0.1$, this enhancement is about $15 \%$, and if $\epsilon=0.3$, it is about $70 \%$. Averaged over all source redshifts, the enhancement of the lensing rate clearly is less. Thus, BATSE's efficiency to detect lensing events is only slightly better than $12 \%$, being perhaps around $15 \%$.

In Figure 5 we show the time delay distributions for the two Monte Carlo simulations presented in Figure 4. The $\epsilon=0$ distribution closely resembles the $\Omega=1, \Lambda=0$ model in Figure 3, except for the absence of a divergence at small $\Delta t$, resulting from the absence of lenses with $L<0.1 L_{*}$ in the simulation. The $\epsilon=0.2$ distribution shows many more short time delay bursts, resulting from images merging across folds. (This distribution includes 
all visible pairs in a multiple image burst. For example, a quadruple burst has six time delays.) The median time delay in this distribution is about three times shorter than in the $\epsilon=0$ distribution. (Both medians here are systematically too large due to the absence of low mass lenses, but if we calibrate the $\epsilon=0$ median with the $0.02 h_{100}^{-1}$ year interval computed from the analytic model, we can get an idea of the true median of the $\epsilon=0.2$ distribution.) Thus, the time delays associated with triple and quadruple imaging may often be less than a day, and most often will be associated with the faintest bursts which are seen only at high magnification.

\section{DISCUSSION AND CONCLUSION}

We have presented three new cosmological models of the GRB population, derived using a double power-law model of burst spectra (TM93), and have computed the rate of double imaging of GRBs by the known population of galaxies, using three different models of galaxy lenses. The highest lensing rate is derived assuming a large cosmological constant and a singular isothermal lens, while the lowest rate has zero cosmological constant and a lens with finite core radius. We predict there should be one lensing event above the BATSE detection threshold every 1.5-25 years. The median time delay between the pair of images is $\sim 0.02$ years. Unfortunately, the instrumental inefficiency associated with BATSE's duty cycle (12\% probability of detecting both components of a double burst) is so severe that seeing a lensing event from a galaxy lens is highly unlikely.

The instrumental inefficiency of observing a lensing event is improved if four components of a burst, such as produced by an elliptical potential, are above the BATSE threshold. In this case there is a $42 \%$ chance of detecting at least two of the four images. Monte Carlo simulations of lensing by elliptical potentials show, however, that quadruple imaging is less common than for quasar lensing, essentially because the amplification bias of lensed bursts is smaller. Consequently, the rate at which lensed bursts will be detected is increased by at most about $20 \%$.

Despite our pessimistic prediction for the detection rate of lensing events, we believe it is well worth the effort to study the BATSE light curves for possible lensing events. A single lensing event, albeit improbable, would verify the cosmological origin of GRBs. Furthermore, even a single detection would suggest one of two exciting possibilities. It might indicate that the universe has a nonzero cosmological constant, giving a larger lensing rate than the "standard" $(\Omega=1, \Lambda=0)$ model. An alternative explanation could be the existence of a significant cosmological density of compact massive lenses (e.g., $10^{6} M_{\odot}$ black holes), in which case the lensing rate could be much higher than for galaxy lensing (Mao 1992b; Blaes \& Webster 1992). The absence of lensed bursts can be used to set constraints on the density of such massive compact objects (Nemiroff et al. 1993).

Nowak and Grossman (1994) have proposed statistical tests for comparing noisy light curves and find that many bursts of comparable duration, but distinct origin can be distinguished by their methods. Their methods are extremely reliable for the $41 \%$ of bursts with $\phi / \phi_{\min } \geq 3$, but for fainter bursts noise often will be sufficient to obscure the differences in the light curves. Thus, consistent pairs of light curves among the brighter bursts 


\section{GRB Lensing}

would be good lensing candidates. Of course, one can only demonstrate that two bursts are different, but not that they have the same source. The frequency of false positives, that is, error measures that are consistent with the lensing hypothesis for two bursts that are actually different, could be investigated by applying the statistical tests to bursts which are known to be distinct (e.g., because they appeared in different parts of the sky). If false positives are very rare, then a pair of consistent light curves may constitute strong evidence of lensing. Calibration of the false positive rate has not been undertaken, however, due to the enormous number of comparisons (thousands) involved. Nowak and Grossman have examined 260 bursts preceding March 5, 1992 in the BATSE catalogue (Fishman et al. 1992), and identified 27 pairs of bursts of comparable duration whose positional error boxes overlap. As expected, there are no candidate lensing events among the bright $\left(\phi / \phi_{\min }\right)$ bursts, and only one fainter statistically consistent pair. However, the duration of this pair of bursts is so short that there are not enough time bins in the light curves for high quality statistical comparison. Thus, to date there are no good lensing candidates in the data.

We emphasize that the improbability of detecting a lensing event is not physical, but mainly instrumental. An experiment designed with lensing in mind could greatly increase the odds of a successful lensing detection. Most obviously, a satellite in a higher orbit could have a duty cycle near 100\%, both because of smaller Earth blockage and because the detectors could operate continuously. Then, instead of $12 \%$ instrumental efficiency to detect both components of a lensed burst, there would be nearly $100 \%$ instrumental efficiency. We also note that three BATSE detectors in a comparable low orbit could also give $100 \%$ efficiency (almost a ten-fold increase in efficiency for three times the cost!).

TM93 have proposed that a "BATSE detector" with 18 times greater sensitivity could detect enough distant bursts in three years to distinguish between $q_{0}=0.1$ and $q_{0}=0.5$ cosmologies. In this case the lensing rate would be about 4 times larger, giving one lensed burst every $0.4-6$ years, with 2.7 years being the mean for the $\Omega=1, \Lambda=0$ cosmology. If GRBs are cosmological in origin, an appropriately designed experiment with high instrumental efficiency would probably detect lensing events. Failure to detect any candidate lensing events within 4.6 times the mean burst interval (say $4.6 \times 2.7 \approx 12$ years) would exclude the cosmological scenario at $99 \%$ confidence.

The authors are indebted to Man Hoi Lee and Chris Thompson for discussions and for pointing out certain references during the course of this project. 


\section{REFERENCES}

Band, D. 1992, ApJ, 400, L63

Band, D., Matteson, J., Ford, L., Schaefer, B., Palmer, C., Teegarden, B., Cline, T., Briggs, M., Paciesas, W., Pendleton, G., Fishman, G., Kouveliotou, Meegan, C., Wilson, R., \& Lestrade, P. 1993, ApJ, 413, 281

Blaes, O.M. 1994, ApJ Suppl, in press,

Blaes, O.M. \& Webster, R.L. 1992, ApJ, 391, L63

Blandford, R.D. \& Narayan, R. 1992, ARA\&A, 30, 311

Carrol, S.M., Press, W.H., \& Turner, E.L. 1992, ARA\&A, 30, 499

Clarke, T.E., Blaes, O., \& Gremaine, S. 1994, AJ, submitted,

Dermer, C.D. 1992, Phys. Rev. Let., 68, 1799

Dyer, C.C. \& Roeder, R.C. 1973, ApJ, 180, L31

Fenimore, E.E., Epstein, R.I., Ho, C., Klebesadel, R. W., Lacey, C. Laros, J.G. Meier, M., Strohmayer, T., Pendleton, G., Fishman, G., Kouvelioutou, C., \& Meegan, C. 1993, Nature, 366, 40

Fishman, G.J., et al. 1992, BATSE Burst Catalog, GROSSC, ,

Fukugita, M., Futamase, T. \& Kasai, M. 1990, MNRAS, 246, 24p

Fukugita, M., Futamase, T., Kasai, M., \& Turner, E.L. 1992, ApJ, 393, 3

Fukugita, M. \& Turner, E.L. 1991, MNRAS, 253, 99 (FT91)

Gehrels, N., Chipman, E., \& Kniffen, D.A. 1993, in Compton Gamma-Ray Observatory, ed. M. Friedlander, N. Gehrels, and D.J. Macomb, American Institute of Physics, p. 3

Gould, A. 1992, ApJ, 386, L5

Grossman, S.A. \& Saha, P. 1994, ApJ, submitted,

Hinshaw, G. \& Krauss, L.M. 1987, ApJ, 320, 468

Huchra, J., Davis, M., Latham, D., Tonry, J. 1983, ApJ Suppl, 52, 89

Lauer, T.R. 1985, ApJ, 292, 104

Lubin, L.M. \& Wijers, R.A.M.J. 1993, ApJ, in press,

Mao, S. 1992a, ApJ, 380, 9

Mao, S. 1992b, ApJ, 389, L41

Mao, S. 1993, ApJ, 402, 382

Mao, S. \& Paczyński, B. 1992a, ApJ, 388, L45

Mao, S. \& Paczyński, B. 1992b, ApJ, 389, L13

Maoz, D. \& Rix, H.-W. 1993, ApJ, 416, 425

Maoz, E. 1993, ApJ, 414, 877

Meegan, C.A., Fishman, G.J., Wilson, R.B., Paciesas, W.S., Pendleton, G.N. Horrack, J.M., Brock, M.N., \& Kouvelioutou, C. 1992, Nature, 355, 143

Narayan, R. and Wallington, R. 1992, in Gravitational Lenses, ed. R. Kayser, T. Schramm, and L. Nieser, Springer-Verlag, p. 12 


\section{6}

GRB Lensing

Narayan, R. and Grossman, S.A. 1989, in Gravitational Lenses, ed. J.M. Moran, J.N. Hewitt, K.Y. Lo, Springer-Verlag, p. 31

Narayan, R. and Wallington, R. 1992a, ApJ, 399, 368

Narayan, R. and Wallington, R. 1992b, in Gravitational Lenses, ed. R. Kayser, T. Schramm, L. Nieser, Springer-Verlag, p. 12

Nemiroff, R.J., Norris, J.P., Wickramasinghe, W.A.D.T., Horrack, J.M., Kouveliotou, C., Fishman, G.J., Meegan, C.A., Wilson, R.B., \& Paciesas, W.S. 1993, ApJ, 414, 36

Norris, J.P., Nemiroff, R.J., Scargle, J.D., Kouvelioutou, C., Fishman, G.J., Meegan, C.A., Paciesas, W.S., \& Bonnell, J.T. 1994, ApJ, in press,

Nowak, M.A. \& Grossman, S.A. 1994, ApJ, submitted,

Ostriker, J.P. \& Vietri, M. 1986, ApJ, 300, 68

Paczyński, B. 1986, ApJ, 308, L43

Paczyński, B. 1987, ApJ, 317, L51

Petrosian, V. 1993, ApJ, 402, L33

Piran, T. 1992, ApJ, 389, L45

Sanitt, N. 1976, MNRAS, 174, 91

Sasaki, S. \& Takahara, F. 1993, MNRAS, 262, 681

Schmidt, M. 1968, ApJ, 151, 393

Tamblyn, P. \& Melia, F. 1993, ApJ, 417, L21 (TM93)

Turner, E.L. 1990, ApJ, 365, L43

Turner, E.L., Ostriker, J.P., \& Gott, J.R. 1984, ApJ, 284, 1

Wallington, S. and Narayan, R. 1993, ApJ, 403, 517

Wambsganss, J. 1993, ApJ, 406, 29

Wickramasinghe, W.A.D.T., Nemiroff, R.J., Norris, J.P., Kouvelioutou, C., Fishman, G.J., Meegan, C.A., Wilson, R.B., \& Paciesas, W.S. 1993, ApJ, 411, L55 
TABLE 1

Cosmological GRB Models

\begin{tabular}{lllll}
\hline \hline$\Omega$ & $\Lambda$ & $L_{0} h_{100}^{2} \mathrm{erg} \mathrm{s}^{-1}$ & $n_{0} h_{100}^{-3} \mathrm{yr}^{-1} \mathrm{Mpc}^{-3}$ & $z_{\max }$ \\
\hline 1.0 & 0.0 & $1.1 \times 10^{50}$ & $7.8 \times 10^{-8}$ & 0.83 \\
0.1 & 0.0 & $2.0 \times 10^{50}$ & $4.2 \times 10^{-8}$ & 0.94 \\
0.1 & 0.9 & $6.0 \times 10^{50}$ & $1.3 \times 10^{-8}$ & 1.13
\end{tabular}

TABLE 2

Cumulative Lensing Rates and Amplification Biases

\begin{tabular}{lccccccc}
\hline \hline & & \multicolumn{2}{c}{ SIS } & \multicolumn{2}{c}{ NSIS } & \multicolumn{2}{c}{ QDEV } \\
$\Omega$ & $\Lambda$ & $N_{l}\left(\mathrm{yr}^{-1}\right)$ & $B$ & $N_{l}\left(\mathrm{yr}^{-1}\right)$ & $B$ & $N_{l}\left(\mathrm{yr}^{-1}\right)$ & $B$ \\
\hline 1.0 & 0.0 & 0.09 & 1.02 & 0.04 & 1.91 & 0.04 & 0.01 \\
0.1 & 0.0 & 0.17 & 0.98 & 0.08 & 0.48 & 0.07 & 0.0005 \\
0.1 & 0.9 & 0.67 & 9.94 & 0.35 & 1.56 & 0.20 & 0.0004
\end{tabular}




\section{GRB Lensing}

\section{FIGURE CAPTIONS}

1. The redshift distribution of GRB as computed from equation (5), with and without correction for incompleteness at faint fluxes near the detection threshold. The curves are labeled by the cosmological parameters $(\Omega, \Lambda)$. The dotted vertical lines indicate the values of $z_{\max }$, which by definition, correspond to $50 \%$ completeness. The integrals under the curves allowing for incompleteness give 800 bursts per year.

2. The differential lensing rate as a function of source redshift $z_{\mathrm{s}}$ for the SIS lens model. The curves are labeled by the cosmological parameters $(\Omega, \Lambda)$, and the cumulative lensing rates $N_{l}$ (i.e., the integrals under the three curves) are given at upper right.

3. The lensing rate as a function of time delay $\Delta t$ for the SIS lens model. The curves are labeled by the cosmological parameters $(\Omega, \Lambda)$, and the cumulative lensing rates $N_{l}$ (i.e., the integrals under the three curves) are given at upper right. The median time delays are about $0.02 h_{100}^{-1}$ years ( 7 days).

4. The rate of multiple imaging by an elliptical potential $(\epsilon=0.2)$, measured by cross section $\sigma_{\epsilon}(2+)$, compared to the rate of double imaging by a circular potential, measured by cross section $\sigma_{0}(2)$, is drawn as the solid, bold line. The multiple imaging cross section is divided into the contributions of double, triple, and quadruple images. (That is, the bottom curve is $\sigma_{\epsilon}(2)$, the next one is $\sigma_{\epsilon}(2)+\sigma_{\epsilon}(3)$, and the next one, $\sigma_{\epsilon}(2+)$, is $\sigma_{\epsilon}(2)+\sigma_{\epsilon}(3)+\sigma_{\epsilon}(4)$.) Double images dominate at low redshift, but triple and quadruple images are increasingly important at higher redshift. Due to the low amplification bias, they never dominate, however. Accounting for BATSE's detection efficiency, we show the enhancement of the detection rate, $N_{\epsilon} / N_{0}$, as the dashed line. The elliptical potential gives an increased detection rate of at most a few tens of per cent. At redshifts $z_{\mathrm{s}} \gtrsim 2.5$, fewer than 10/10000 sources in the Monte Carlo simulation have observable multiple images, and numerical noise dominates the results.

5. This distribution of time delays between pairs of images derived from the Monte Carlo simulation for $\epsilon=0$ (solid histogram) and $\epsilon=0.2$ (dashed histogram). The curves should be compared to the $\Omega=1, \Lambda=0$ model in Fig. 3. The curves do not diverge at small $\Delta t$ because of the absence of lensing galaxies fainter than $L=0.1 L_{*}$ in the simulation. Images merging across folds produce many more short time delays in the $\epsilon=0.2$ model than in the $\epsilon=0$ model, giving a median time delay about three times shorter. 
Figure 1 
20 GRB Lensing

Figure 2 
GRB Lensing $\mathbf{2 1}$

Figure 3 
22 GRB Lensing

Figure 4 
GRB Lensing $\mathbf{2 3}$

Figure 5 
This figure "fig1-1.png" is available in "png" format from: http://arxiv.org/ps/astro-ph/9401047v2 
This figure "fig1-2.png" is available in "png" format from: http://arxiv.org/ps/astro-ph/9401047v2 